\title{
Analysis of strain relatedness using High Resolution Melting in a case of recurrent candiduria
}

Sara Gago ${ }^{1}$, Belen Lorenzo ${ }^{2}$, Alicia Gomez-Lopez ${ }^{1}$, Isabel Cuesta ${ }^{3}$, Manuel Cuenca-Estrella ${ }^{1}$ and Maria J Buitrago ${ }^{1 *}$

\begin{abstract}
Background: Several genotyping protocols have been described to study Candida albicans strains with different sensitivity values. In this study we have analyzed the genetic relatedness and the antifungal susceptibility of several Candida albicans strains isolated from a patient who from suffered recurrent candiduria for a period of five years. Strains were genotyped using Microsatellite Length Polymorphism (MLP) with three microsatellite markers (HIS 3, EF 3 and CDC 3), and a new method based on high resolution melting (HRM) was developed to analyze the microsatellite region. This method was compared with the conventional technique that uses capillary electrophoresis.

Results: MICs of the isolates showed the existence of fluconazole susceptible and resistant strains. An inter-colony test using single concentration ( 8 and $16 \mathrm{mg} /$ ) of fluconazole revealed the coexistence of both fluconazole susceptible and resistant strains. Both genotyping analysis methods showed that all the patient's isolates had a clonal origin. HRM analysis method developed was able to accurately establish strain relatedness and presented a discriminatory power of 0.77 .

Conclusions: Although HRM analysis method presented a lower discriminatory power compared to methods based on capillary electrophoresis, it provided a more cost-effective and suitable alternative for genotyping $C$. albicans in a clinical laboratory.
\end{abstract}

Keywords: Candiduria, High resolution melting, Genotyping

\section{Background}

Candida albicans is a ubiquitous commensal in healthy individuals; it is, however, a very important opportunistic pathogen for immunologically weak and immunocompromised people [1]. Recurrent and/or persistent infections by Candida species are frequent, particularly in oropharyngeal and vaginal candidiasis, although it has also been described in urinary tract infections [2]. Studies describing recurrent infections have focused on determining the relatedness between colonizing and infective strains [3,4], as well as between successive infective strains [5-9]. It seems clear now that the majority of commensal and infecting populations of C. albicans

\footnotetext{
* Correspondence: buitrago@isciii.es

'Servicio de Micología, Centro Nacional de Microbiología, Instituto de Salud Carlos III, Ctra Majadahonda-Pozuelo, Km 2, Majadahonda, Madrid 28220, Spain

Full list of author information is available at the end of the article
}

from the same individuals are clonal in origin but subsequently undergo microevolution at the site of colonization and through recurrent episodes of infection $[5,10,11]$. The microevolution of the strains is a frequent process in recurrent infections and it takes place in response to adaptive changes $[9,12]$. A recent work which examined the "in vitro" dynamics of $C$. albicans populations in the presence or absence of fluconazole has shown that mutations that lead to increased drug resistance appear frequently [13]. Others authors suggest that natural C. albicans populations comprise a mixture of closely related strain types [6].

Typing methods have been described as useful tools for the differentiation between strains isolated only once and those able to cause recurrent infections. Although several typing methods have been described for C. albicans (AFLP, RFLPPCR or MLST), one of the most suitable is the fragment length analysis of microsatellites called Microsatellite Length

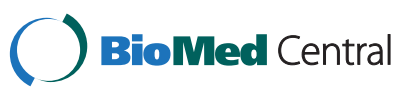


Polymorphism (MLP). This technique has a high discriminatory power and reproducibility. MLP analysis has proved its efficacy and reproducibility in a large number of epidemiological studies [9,14-19]; however, this technique is not easy to use and the estimated cost per isolate remains high.

The High Resolution Melting (HRM) provides a faster and cheaper method for microsatellite fragment analysis. This technique uses fluorescent DNA binding dyes with improved saturation properties allowing a precise assessment of sequence variation based on DNA melting curves analysis $[20,21]$. The suitability of HRM to discriminate PCR products based on one nucleotide change has also been described. Some recent articles, focusing on the capacity of HRM to identify and genotype fungi, have been reported [15,22].

In this work, we developed a method based on HRM to assess the relatedness of strains in a clinical case of recurrent candiduria. The results were compared with the conventional MLP genotyping techniques. The isolates, recovered over a period of five years, additionally showed significant differences in their susceptibility to antifungal agents. Antifungal susceptibility test and selection of resistant population was performed.

\section{Methods}

\section{Origin of the strains and clinical data from the patient}

The strains were isolated from a 62 year old male with medullary sponge right kidney (Carchi-Ricci disease) and recurrent reno-urethral lithiasis subjected to several lithotripsies. The patient was admitted in a Tertiary General Hospital (Hospital Virgen de la Concha, Zamora, Spain) diagnosed with right pyelonephritis caused by obstructive kidney stones. C. albicans was isolated in blood cultures and urocultures. The antifungal susceptibility profile showed that azoles and amphotericin B were active in vitro against this strain (CNM-CL-4929, Yeast Collection of the Spanish National Centre for Microbiology) as shown in Table 1.

Treatment with ciprofloxacin $400 \mathrm{mg} / 12 \mathrm{~h}$ and fluconazole iv $200 \mathrm{mg} / 12 \mathrm{~h}$ was started. After three days of treatment, as fever persisted and blood and urine cultures remained positive, fluconazole was replaced by amphotericin B lipid complex $200 \mathrm{mg} / 24 \mathrm{~h}$ iv and $100 \mathrm{mg}$ every other day. Six days after admission, lithotripsy was performed and a double $J$ stent was placed. He was discharged from hospital a month after admission. From 2003 to 2008, the patient suffered from several episodes of Candida infection and underwent multiple lithotripsies. He was treated with oral fluconazole $(200 \mathrm{mg} / 12 \mathrm{~h})$ several times. A total of 18 strains were isolated from urine and sent to the Mycology Reference Laboratory for identification and susceptibility testing (Table 1). In 2006 the susceptibility of one of the isolates (CNM-CL-6188) showed high MICs for fluconazole ( $>8 \mathrm{mg} / \mathrm{l}$ ). Three months later, the patient was readmitted to the hospital and diagnosed with hydronephrosis and

Table 1 MICs values and isolation data of the clinical isolates used in this study

\begin{tabular}{|c|c|c|c|c|c|c|c|c|c|c|c|c|}
\hline Strain & Isolate origin & Isolation data & $A B$ & $5 F Z$ & $F Z$ & IZ & VZ & $\mathrm{PZ}$ & $\mathrm{CA}$ & MC & AN & \\
\hline CNM-CL-4929 & Blood culture & 02-28-2003 & 0.03 & 0.12 & 0.12 & 0.015 & 0.015 & * & * & $*$ & * & \\
\hline L06/31 & Urine & 02-01-2006 & 0.12 & 0.12 & 0.5 & 0.015 & 0.015 & 0.015 & 0.03 & 0.03 & 0.03 & \\
\hline L06/32 & Urine & $02-01-2006$ & 0.12 & 0.12 & 0.5 & 0.015 & 0.015 & 0.015 & 0.03 & 0.03 & 0.03 & \\
\hline CNM-CL-6188\# & Urine & 08-18-2006 & 0.25 & 0.12 & $>64$ & $>8$ & $>8$ & $>8$ & 0.25 & 0.03 & 0.03 & \\
\hline L06/260 & Urine & 08-18-2006 & 0.06 & 0.12 & 8 & 1 & 0.12 & 1 & 0.03 & 0.03 & 0.03 & \\
\hline L06/349 & Urine & 11-07-2006 & 0.06 & 0.12 & 0.25 & 0.015 & 0.015 & 0.015 & 0.12 & 0.03 & 0.03 & \\
\hline L06/350 & Urine & $11-07-2006$ & 0.06 & 0.12 & 0.25 & 0.015 & 0.015 & 0.015 & 0.12 & 0.03 & 0.03 & \\
\hline CNM-CL-6361"\# & Urine & 03-20-2006 & 0.25 & 0.12 & $>64$ & $>8$ & $>8$ & $>8$ & 0.25 & 0.03 & 0.03 & \# \\
\hline CNM-CL-6373\# & Urine & 04-16-2006 & 0.12 & 0.12 & $>64$ & 1 & $>8$ & 4 & 0.25 & 0.03 & 0.03 & \\
\hline L07/130 & Urine & 04-16-2006 & 0.12 & 128 & 16 & 16 & 16 & 16 & 0.25 & 0.03 & 0.03 & \\
\hline CNM-CL-6399\# & Urine & 05-21-2007 & 0.25 & 0.12 & $>64$ & $>8$ & $>8$ & $>8$ & 0.25 & 0.03 & 0.03 & \# \\
\hline CNM-CL-6431" & Urine & 06-13-2007 & 0.25 & 0.12 & $>64$ & $>8$ & $>8$ & $>8$ & 0.25 & 0.03 & 0.03 & \# \\
\hline CNM-CL-6488\# & Urine & 07-27-2007 & 0.12 & 0.12 & 0.25 & 0.015 & 0.015 & 0.015 & 0.25 & 0.03 & 0.03 & \# \\
\hline L07/453 & Urine & $11-21-2007$ & 0.03 & 0.12 & 0.12 & 0.015 & 0.015 & 0.015 & 0.12 & 0.03 & 0.03 & \\
\hline L07/454 & Urine & $11-21-2007$ & 0.03 & 0.12 & 0.25 & 0.06 & 0.06 & 0.12 & 0.06 & 0.03 & 0.03 & \\
\hline CNM-CL-6714\# & Urine & 03-07-2008 & 0.25 & 0.12 & $>64$ & 0.06 & $>8$ & $>8$ & 0.25 & 0.03 & 0.03 & \# \\
\hline CNM-CL-7019\# & Urine & $11-12-2008$ & 0.12 & 0.12 & 2 & 0.12 & 0.015 & 0.06 & 0.25 & 0.03 & 0.03 & \# \\
\hline CNM-CL-7020\# & Urine & $11-12-2008$ & 0.25 & 0.12 & 0.25 & 0.015 & 0.015 & 0.015 & 0.25 & 0.03 & 0.03 & \# \\
\hline
\end{tabular}

\# Strains included in the genotyping analysis.

* No MICs data available.

CNM-CL Yeast Collection of the Spanish National Center for Microbiology. 
kidney obstruction after the placement of a double J stent in the right kidney. The stent was removed and a percutaneous nephrostomy was performed. An initial treatment with caspofungin $70 \mathrm{mg}$ iv and then $50 \mathrm{mg}$ iv $/ 24 \mathrm{~h}$ was established. Later, the patient was treated with amphotericin B lipid complex (11 days) and was subsequently put back on caspofungin until patient was discharged from the hospital. During the stay in the hospital, blood cultures were negative while urine cultures remained positive until the patient was treated with amphotericin B. The patient's isolates were controlled in an outpatient mode up to the end of 2008, at which time the patient went to another institution and no more samples were taken. The written informed consent was sought and obtained from the patient according to Spanish regulations at that date. The patient also signed his consent to the release of his clinical and personal information in a scientific publication.

\section{Antifungal susceptibility testing}

Antifungal susceptibilities were tested in vitro according to the EUCAST microdilution method (AFST-EUCAST, definitive document 7.1). Interpretative breakpoints proposed by EUCAST for fluconazole and voriconazole were used [23]. For the rest of the antifungal tested, the breakpoints proposed by Rodriguez-Tudela et al. were used [24]. The antifungal agents used were amphotericin B, flucytosine, fluconazole, itraconazole, voriconazole, posaconazole, caspofungin, micafungin, and anidulafungin. Isolates were stored at $-20^{\circ} \mathrm{C}$ until use.

\section{Selection of resistant population}

In February of 2011, the isolates available in our culture collection (Tables 1 and 2) were subcultured for genotyping studies. To analyze the probability of the coexistence of fluconazole resistant and susceptible populations in each isolate, we performed a screening assay based on a singleconcentration fluconazole test [25]. The antifungal concentration used in this assay was selected on the basis of the MIC values previously obtained. The test of growth was performed in microplates containing RPMI 1640 medium supplemented with $2 \%$ glucose (Sigma-Aldrich, Madrid, Spain) and a final fluconazole concentration of 8 and $16 \mathrm{mg} / \mathrm{l}$. Ten colonies of each isolate were tested. For each colony, a suspension of $10^{5} \mathrm{cfu} / \mathrm{ml}$ was prepared. Plates were inoculated with $0.1 \mathrm{ml}$ from the cell suspension. A growth control was also included. The Optical Density (OD) at $530 \mathrm{~nm}$ was measured after 24 and 48 hours of incubation. The reduction of the OD below 50\% compared to control was considered as susceptibility to fluconazole.

\section{Genotyping studies}

Nine representative strains isolated from the patient on different days were selected for performance of genotyping studies (Tables 1 and 3). The control population
Table 2 Intercolony fluconazole susceptibility in single concentration microdilution plates

\begin{tabular}{ccc}
\hline & \multicolumn{2}{c}{ No of colonies fluconazole resistant } \\
\cline { 2 - 3 } Strain & $\mathbf{8} \mathbf{~ m g ~ / I}$ & $\mathbf{1 6} \mathbf{~} \mathbf{g} / \mathbf{l}$ \\
\hline CNM-CL-6188 & $2 / 10$ & $1 / 10$ \\
CNM-CL-6361 & $5 / 10$ & $4 / 10$ \\
CNM-CL-6373 & $9 / 10$ & $9 / 10$ \\
CNM-CL-6399 & $10 / 10$ & $4 / 10$ \\
CNM-CL-6431 & $2 / 10$ & $2 / 10$ \\
CNM-CL-6488 & $0 / 10$ & $0 / 10$ \\
CNM-CL-6714 & $4 / 10$ & $4 / 10$ \\
CNM-CL-7019 & $0 / 10$ & $0 / 10$ \\
CNM-CL-7020 & $0 / 10$ & $0 / 10$ \\
\hline
\end{tabular}

CNM-CL Yeast Collection of the Spanish National Center for Microbiology.

consisted of 20 strains from patients geographically and temporally unrelated. Nineteen out of 20 isolates were from whole blood and the remaining isolate was from pleural fluid (Table 3). ATCC64548 and ATCC64550 C. albicans reference strains were also included in this study. All isolates were identified by physiological and morphological tests, including microscopic examination and biochemical tests. The identification was confirmed by sequence analysis of the ITS (internal transcribed spacer) region of the rDNA [26].

Yeast cells were grown for 24 hours in Sabouraud broth medium at $30^{\circ} \mathrm{C}$. Genomic DNA was extracted using a phenol:chloroform method [27] followed by purification using Chroma SPIN + TE 400 columns according to the manufacturer's instructions (Clontech Laboratories, Becton Dickinson, Madrid, Spain).

Genotyping analysis of $C$. albicans was performed using MLP procedure with three different markers previously described, CDC 3 [28]; EF 3 [29] and HIS 3 [30]. The primers sequences used to amplify these markers were: $\mathrm{CDC} 3$ forward (5' CAGATGATTTTTTGTATGAGAAGAA3 ${ }^{\prime}$ ) and reverse (5' CAGTCACAAGATTAAAATGTTCAAG3'); EF3 forward (5 TTTCCTCTTCCTTTCATATAGAA3') and reverse (5 GGATTCACTAGCAGCAGACA3') and HIS3 forward (5 TGGCAAAAATGATATTCCAA3') and reverse (5' TACACTATGCCCCAAACACA $3^{\prime}$ ).

MLP analysis using capillary electrophoresis was modified from Botterel et al. [14]. Alleles were amplified in a multiplex PCR in a $50 \mu \mathrm{l}$ final volume containing 20 ng DNA, 1X PCR-Buffer II (Applied Biosystems, Madrid, Spain), $0.2 \mathrm{mM}$ of each deoxynucleotide triphosphate, $5 \mathrm{mM}$ of $\mathrm{MgCl}_{2}$, and $0.15 \mu \mathrm{M}$ of each primer and $1 \mathrm{U}$ of AmpliTaq Polymerase (Applied Biosystems).

Sense CDC3 primer was labelled with 4, 7, 2', 4', 5', $7^{\prime}$ hexachloro-6-carboxyfluorescein (HEX), EF3 antisense primer with 6-carboxyfluorescein (FAM) and HIS 3 
Table 3 Microsatellite lenght (bp) for the three microsatellite markers using capillary electrophoresis

\begin{tabular}{|c|c|c|c|c|}
\hline \multirow{2}{*}{ Strain } & \multirow{2}{*}{$\begin{array}{l}\text { Isolate } \\
\text { origin }\end{array}$} & \multicolumn{3}{|c|}{ Length (bp) determined by PCR analysis of microsatellite markers: } \\
\hline & & CDC 3 & EF 3 & HIS 3 \\
\hline CNM-CL-7426 ${ }^{\mathrm{a}}$ & Whole blood & $117 / 125$ & $125 / 125$ & $162 / 186$ \\
\hline CNM-CL-7449 & Whole blood & $117 / 125$ & $125 / 125$ & $162 / 190$ \\
\hline CNM-CL-7470 ${ }^{\mathrm{a}}$ & Whole blood & $117 / 125$ & $120 / 120$ & $162 / 227$ \\
\hline CNM-CL-7471 ${ }^{\mathrm{a}}$ & Whole blood & $117 / 117$ & $130 / 130$ & $162 / 162$ \\
\hline CNM-CL-7478 & Whole blood & $117 / 125$ & $120 / 120$ & $202 / 202$ \\
\hline CNM-CL-7484 & Whole blood & $125 / 125$ & $125 / 125$ & $162 / 190$ \\
\hline CNM-CL-7498 & Whole blood & $125 / 129$ & $130 / 139$ & $149 / 166$ \\
\hline CNM-CL-7499 & Whole blood & $117 / 129$ & $130 / 139$ & $154 / 154$ \\
\hline CNM-CL-7503 & Whole blood & $117 / 117$ & $126 / 138$ & $153 / 182$ \\
\hline CNM-CL-7504 & Whole blood & $117 / 117$ & $124 / 130$ & $149 / 166$ \\
\hline CNM-CL-7513 & Whole blood & $121 / 125$ & $124 / 137$ & $158 / 158$ \\
\hline CNM-CL-7617 & Whole blood & $117 / 117$ & $124 / 130$ & $313 / 313$ \\
\hline CNM-CL-7624 & Whole blood & $117 / 117$ & $126 / 138$ & $153 / 153$ \\
\hline CNM-CL-7620 & Whole blood & $117 / 125$ & $120 / 120$ & $162 / 210$ \\
\hline CNM-CL-7640 & Whole blood & $125 / 129$ & $130 / 137$ & $149 / 166$ \\
\hline CNM-CL-7643 & Pleural fluid & $117 / 117$ & $124 / 130$ & $149 / 166$ \\
\hline CNM-CL-7683 & Whole blood & $117 / 125$ & $120 / 129$ & $162 / 210$ \\
\hline CNM-CL-7694 & Whole blood & $117 / 129$ & $130 / 139$ & $148 / 153$ \\
\hline CNM-CL-7705 ${ }^{a}$ & Whole blood & $117 / 117$ & $124 / 130$ & $-1-$ \\
\hline CNM-CL-7712a & Whole blood & $117 / 125$ & $120 / 129$ & $162 / 210$ \\
\hline ATCC64548 & Whole blood & $113 / 113$ & $124 / 124$ & $162 / 162$ \\
\hline ATCC64550 & Whole blood & $117 / 125$ & $120 / 129$ & $162 / 178$ \\
\hline CNM-CL-6188 ${ }^{b}$ & Urine & $121 / 121$ & $127 / 129$ & $153 / 153$ \\
\hline CNM-CL-6361 ${ }^{\text {b }}$ & Urine & $121 / 121$ & $127 / 129$ & $153 / 153$ \\
\hline CNM-CL-6373 ${ }^{b}$ & Urine & $121 / 121$ & $127 / 129$ & $153 / 153$ \\
\hline CNM-CL-6399 & Urine & $121 / 121$ & $127 / 129$ & $153 / 153$ \\
\hline CNM-CL-6431 ${ }^{\mathrm{b}}$ & Urine & $121 / 121$ & $127 / 129$ & $153 / 153$ \\
\hline CNM-CL-6488 & Urine & $121 / 121$ & $127 / 129$ & $153 / 153$ \\
\hline CNM-CL-6714 & Urine & $121 / 121$ & $127 / 129$ & $153 / 153$ \\
\hline CNM-CL-7019 & Urine & $121 / 121$ & $127 / 129$ & $153 / 153$ \\
\hline CNM-CL-7020 & Urine & $121 / 121$ & $127 / 129$ & $153 / 153$ \\
\hline
\end{tabular}

CNM-CL Yeast Collection of the Spanish National Center for Microbiology. a: Control population.

b: strains from the case study included for genotyping studies.

sense primer was labelled with $2^{\prime}$-chloro- $5^{\prime}$-fluoro- $7^{\prime}, 8^{\prime}$ fused phenyl-1.4-dichloro-6-carboxyfluorescein (NED). Primers were synthesized by Sigma-Aldrich (SigmaAldrich, Madrid, Spain).

PCR reactions were performed in a GeneAmp PCR system 9700 (Applied Biosystems). The cycling conditions included a first step for preincubation (activation of the enzyme) and denaturation of the DNA template at $95^{\circ} \mathrm{C}$ during 5 minutes. Next steps consisted in an amplification program of 30 cycles as follow: denaturation at $95^{\circ} \mathrm{C}$ for $30 \mathrm{~s}$, annealing at $55^{\circ} \mathrm{C}$ for $30 \mathrm{~s}$ and extension at $72^{\circ} \mathrm{C}$ for $1 \mathrm{~min}$ with a final extension step of $7 \mathrm{~min}$ at $72^{\circ} \mathrm{C}$.

To assess the size of the fragments, $1 \mu \mathrm{l}$ of the PCR products was added to $9 \mu \mathrm{l}$ of Formamide $\mathrm{Hi}-\mathrm{Di}$ (Applied Biosystems, Madrid, Spain) and $1 \mu \mathrm{l}$ of the internal size standard ROX 500 (Applied Biosystems, Madrid, Spain). Capillary electrophoresis was run using the ABI 3730 XL (Applied Biosystems, Madrid, Spain) sequencer. Fragment size for the different alleles was calculated with GeneMapper version 3.0 (Applied Biosystems, Madrid, Spain). 
A

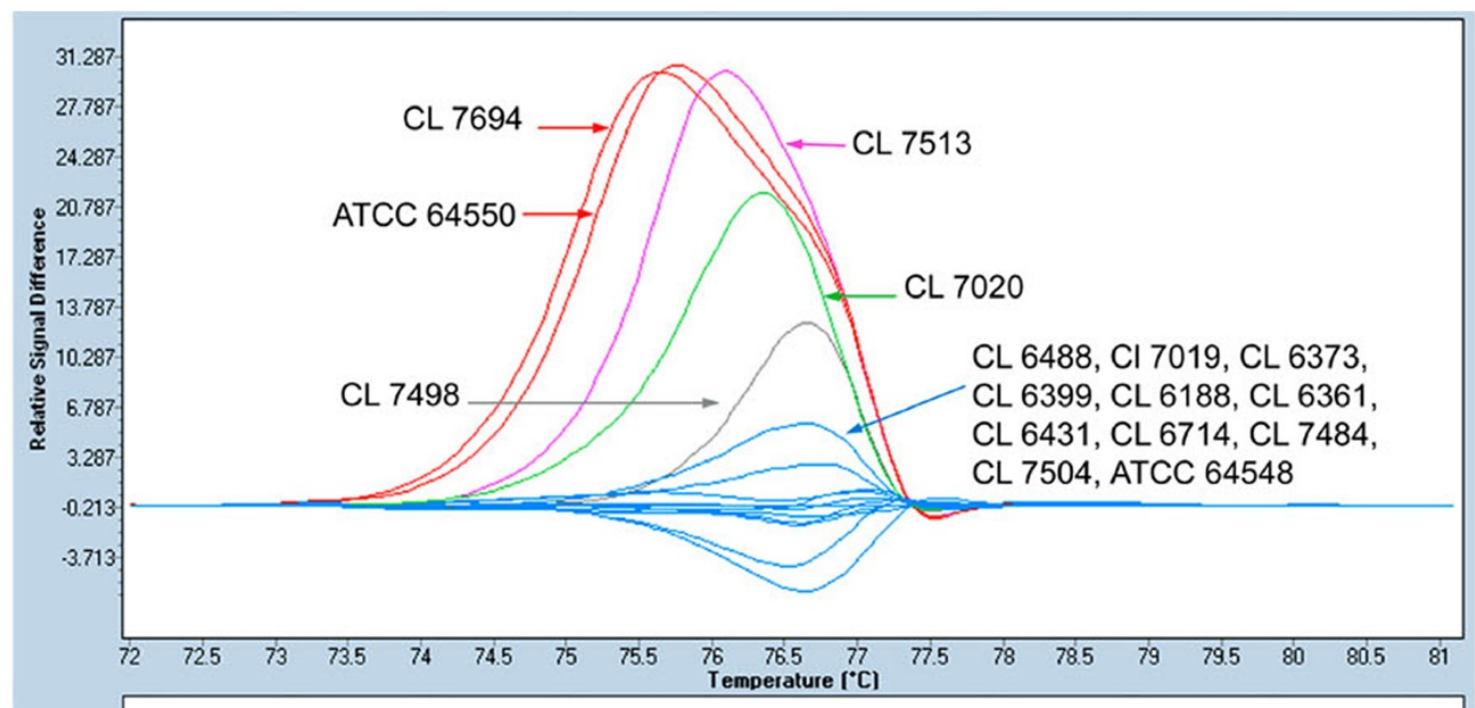

B

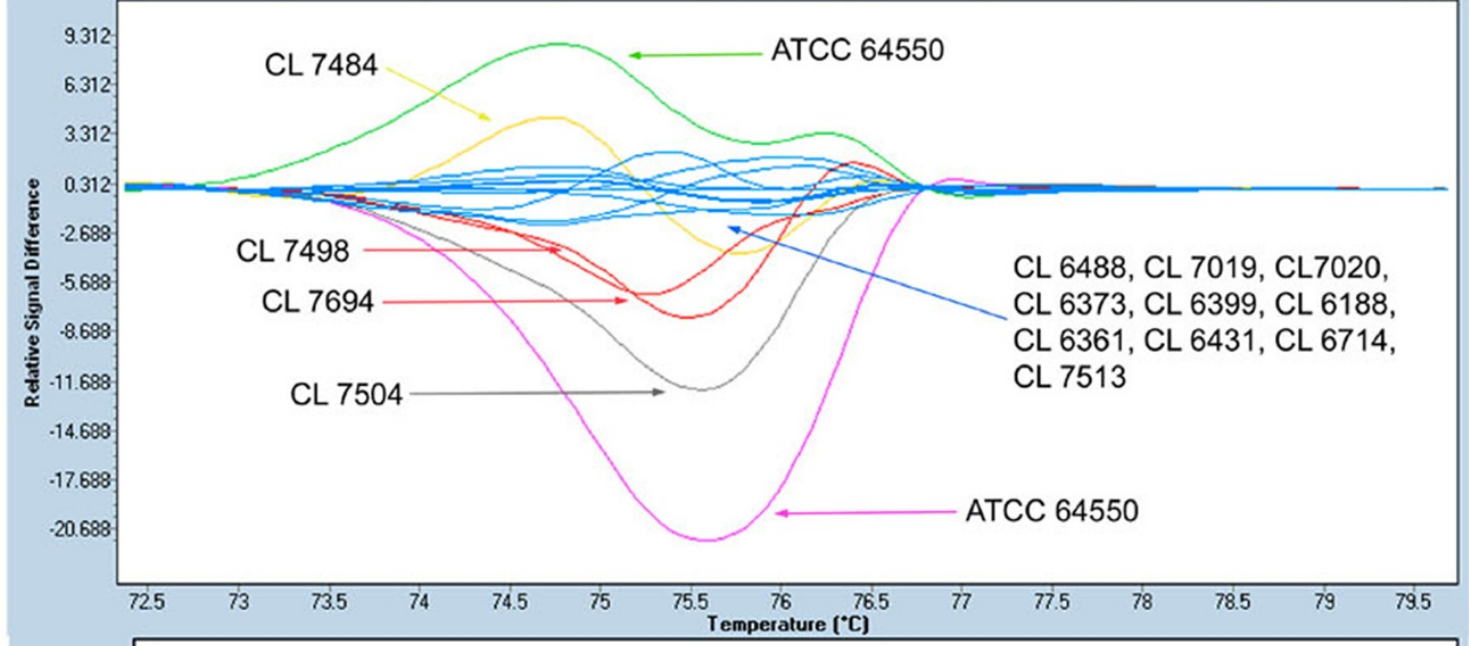

C

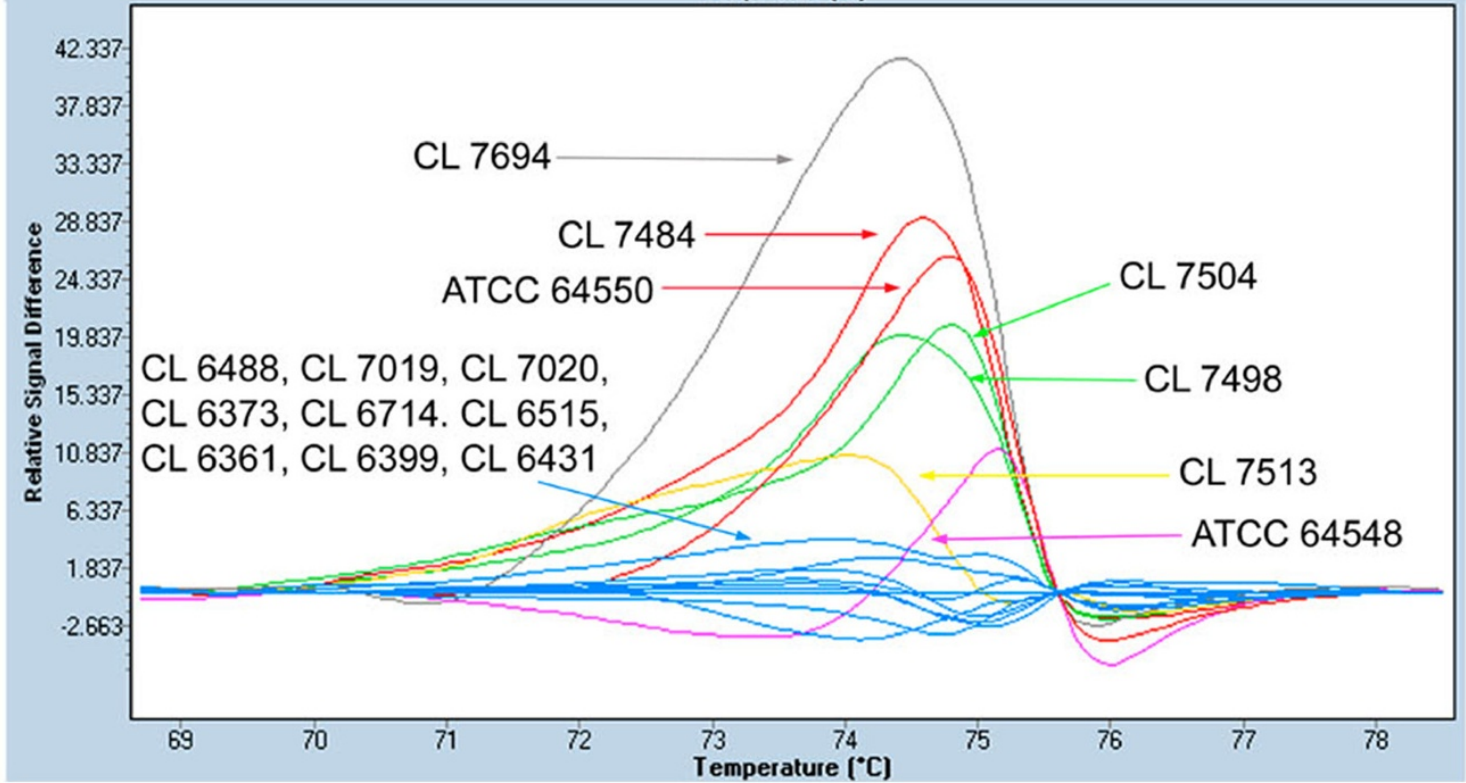

Figure 1 (See legend on next page.) 
In addition, a HRM-based analysis was performed using singleplex PCRs with each pair of primers without any modification of the reaction conditions. Control population was selected based on MLP results. Strains included as control were: CL 7484, CL 7498, CL 7504, CL 7513, CL 7694, ATCC 64548 and ATCC 64550 (Figure 1). Seven different genotypes for the three markers were chosen (Figure 1).

After PCR, HRM analysis was performed in a LightCycler 480 system (Roche, Madrid, Spain). To obtain the HRM curves, $1 \mu \mathrm{l}$ of LightCycler ${ }^{\circledR} 480$ ResoLight Dye (Roche, Madrid, Spain) was added to PCR products and the reactions were incubated at $95^{\circ} \mathrm{C} 1 \mathrm{~min}$, followed by a renaturation step of $40^{\circ} \mathrm{C}$ for $1 \mathrm{~min}$. Melting curves were generated by ramping from $65^{\circ}$ to $95^{\circ}$ at $0.02^{\circ} \mathrm{C} / \mathrm{s}, 25$ acquisitions $/{ }^{\circ} \mathrm{C}$. HRM curves were plotted using the automated grouping option provided by the software and by manual editing for each microsatellite marker. Normalization conditions for each microsatellite marker are shown in Table 4 .

For sequencing, amplicons were treated with ExoSap IT (GE Health Care, Madrid, Spain) following the manufacturer's instructions. Sequencing reactions were performed in a GeneAmp PCR system 9700 (Applied Biosystems). Sequences were analyzed in triplicate.

The numerical index of discriminatory power for each marker and for the multiplex analysis was calculated in both genotyping analysis using the Simpson biodiversity index (D) [31]. The percentage of heterozygosis has been calculated by the ratio number of heterozygous genotypes/ total number of genotypes.

\section{Results}

\section{Antifungal susceptibility testing}

Antifungal susceptibility results are shown in Table 1. At first, isolates were susceptible to all antifungal agents tested; however, in August 2006 an isolate showed an azole-resistant phenotype and subsequently isolates susceptible and resistant to azoles appeared at random. Between March 2006 and June 2007 all strains tested were azole-resistant but this pattern changed again between
July and November 2007. The latest azole resistant strain recovered was from March 2008.

\section{Fluconazole resistance selection}

Ten colonies of each of the nine isolates genotyped were tested for fluconazole resistance at 8 and $16 \mathrm{mg} / \mathrm{l}$ final concentration. From five out of the 9 strains we were able to select resistant and susceptible isolates. On the other hand, from one strain all colonies were resistant and from the remaining three strains all checked colonies were susceptible to fluconazole in a final concentration of $8 \mathrm{mg} / \mathrm{l}$. When fluconazole concentration was increased to $16 \mathrm{mg} / \mathrm{l}$, the number of resistant colonies was reduced (Table 2).

\section{Genotyping studies}

\section{Microsatellite length genotyping}

Microsatellite markers were used to genotype the nine strains recovered from the patient. Each PCR product was assigned to an allele [14] so each strain was characterized by 6 alleles that were differently coupled (Table 3). Strains from the patient showed the same microsatellite pattern for the three markers and they were different from the control population (Table 3). All the isolates recovered from the patient were homozygous for CDC 3 and HIS 3 markers while they showed a heterozygous genotype for EF 3 (Table 5).

The $\mathrm{D}$ value for EF3 was 0.86 , similar to that previously reported [14,15], for CDC 3 it was 0.81 , and for HIS 3 it was 0.87 . The combination of three markers yielded a discriminatory power of 0.92 (Table 5).

\section{Microsatellite HRM genotyping}

When analysis by HRM was performed, isolates from the patient were grouped together for the three markers analyzed (Figure 1).

The analysis of marker CDC 3 showed that all homozygous strains, including those from the patient, were plotted in one group except for the CNM- CL 7020 strain (Figure 1A). Due to the unexpected result for CNM-CL7020, the PCR product was sequenced (6x

Table 4 High resolution melting conditions and discrimination power

\begin{tabular}{lllll}
\hline HRM Marker & Pre-Melt T & Post-Melt T & Threshold/Sensitivity & DP \\
\hline CDC 3 & $71.95-72.96$ & $79.34-81.11$ & $5.645 / 0.3$ & 0.53 \\
EF 3 & $70.8-72.62$ & $78.46-79.71$ & $5.645 / 0.3$ & 0.62 \\
HIS 3 & $68.65-69.82$ & $77.42-78.56$ & $5.645 / 0.3$ & 0.68 \\
Multiplex & & & & 0.77 \\
\hline
\end{tabular}


Table 5 Characteristics of the microsatellite loci analyzed by capillary electrophoresis

\begin{tabular}{lccccc}
\hline Microsatellite Marker & No. of alleles & No. of genotypes & No. of heterozygotic genotypes & DP & \% heterozygosity \\
\hline CDC 3 & 5 & 8 & 4 & 0.81 & 50.00 \\
EF 3 & 10 & 11 & 7 & 0.86 & 63.63 \\
HIS 3 & 14 & 15 & & 0.88 & 53.30 \\
Multiplex & & & 0.92 & \\
\hline
\end{tabular}

sequence coverage) and a $3 \mathrm{bp}$ insertion at $67 \mathrm{pb}$ from the forward primer was found. Heterozygous strains were distributed in four groups according to their fragment length. The heterozygous strains CNM-CL 7694 and ATCC 64550 were plotted together although one of the alleles were different (Table 3). When we performed EF 3 fragments analysis by HRM, six different groups were plotted one of them contained strains from the patient while the control population was distributed into five groups according to its fragment size or whether they were homozygous or heterozygous (Figure 1B).

Finally, HRM analysis of the HIS3 marker showed six different groups. Strains from the patient were grouped together again. Strains in the control population were grouped based on their fragment size pattern (Figure 1C).

Discrimination power for CDC 3 marker was 0.53 , for EF 3 it was 0.62 and for the HIS 3 marker it was 0.68 . The combination of the three markers provided a DP value of 0.77 (Table 4).

\section{Discussion}

Typing methods have been described as useful tools for the differentiation between strains isolated only once and those able to cause recurrent infections. Several methods have been developed to analyze microevolution and structure of $C$. albicans species. Although MLST (MultiLocus Sequence Typing) has been chosen as the most discriminatory technique [5,32], several articles have recently pointed towards the suitability of MLP [14-16,29]. In this study, nine isolates from a case of recurrent urinary infection were genotyped using microsatellites and a new HRM analysis method. Antifungal susceptibility testing revealed that strains from the patient were susceptible and resistant in vitro to fluconazole in a random way. Microvariation between colonies due to exposure of $C$. albicans to azole antifungal agents has been widely described $[10,16]$ and the need to perform intercolony assays has also been reported [25,33,34]. We performed an inter-colony test modified from Schoofs et al. [25] and we were able to prove the coexistence of colonies resistant and susceptible to azoles in a high number of the strains tested. The number of azole-resistant colonies was variable depending on azole concentration.

A genotyping method based on HRM analysis was developed taking into account previous works showing that if the number of genotypes is higher than seven, the curve definition is not the best possible [35]. Based on that premise, for each marker we selected seven strains with different genotype, previously analysed by capillary electrophoresis. C. albicans microsatellites (CDC3, EF3 and HIS3) were amplified using LightCycler ${ }^{\circledR} 480$ ResoLight as intercalating dye. When HRM analysis was performed, the isolates from the patient were plotted together and these results were consistent with those obtained by capillary electrophoresis. There was only one exception for the CDC3 marker where one strain (CNM-CL7020) was not grouped, as expected, with the other strains showing the same MLP genotype. The sequence of the fragment showed a 3 bp insertion that explained the melting differences. This fact supports previous works in which HRM allowed to identify changes in the sequence length and one nucleotide changes [36].

Although the calculated discrimination power was higher for the analysis using capillary electrophoresis than for HRM analysis (0.92 vs. 0.77) as previously reported [14]. The HRM analysis showed several advantages; it was a very simple and fast technique and results were obtained in 3 hours (including amplification), the interpretation of results was easy and the cost per sample was much lower than MLP genotyping due to this technique does not require sequencing equipment and the primers are not end-labelled. Our estimate is that the cost per sample using capillary electrophoresis is more than twice that of using HRM analysis. Furthermore, it can be used in a routine laboratory setting as it only requires real time PCR equipment. In this study, although we were not able to demonstrate the mechanism underlying the variability in the susceptibility to azoles in the strains tested, we were able to confirm that resistant and susceptible isolates were genetically closely related with an easy method to analyze microsatellites. The results highlight the need for more in-depth studies to be performed on these kinds of infections for an accurate and appropriate management thereof.

\section{Conclusions}

This method is a useful tool for performing a fast screening to establish relatedness between strains in outbreaks or surveillance studies in cases of recurrent or persistent infections. To our knowledge, this is the first study in which three microsatellite markers were analyzed by HRM by using seven strains with different 
genotype as control population and reaching HRM resolution limits. Although HRM analysis method presented a lower degree of discrimination compared to other genotyping methods, it provided a more cost-effective and suitable alternative for genotyping $C$. albicans in a clinical laboratory.

\section{Competing interest}

In the past 5 years, M.C.E. has received grant support from Astellas Pharma, bioMerieux, Gilead Sciences, Merck Sharp and Dohme, Pfizer, Schering Plough, Soria Melguizo SA, the European Union, the ALBAN program, the Spanish Agency for International Cooperation, the Spanish Ministry of Culture and Education, The Spanish Health Research Fund, The Instituto de Salud Carlos III, The Ramon Areces Foundation, The Mutua Madrileña Foundation. He has been an advisor/consultant to the Panamerican Health Organization, Gilead Sciences, Merck Sharp and Dohme, Pfizer, and Schering Plough. He has received remuneration for talks on behalf of Gilead Sciences, Merck Sharp and Dohme, Pfizer, and Schering Plough.

\section{Authors' contributions}

SG performed the genotyping studies, the analysis of the results and also participated in drafting the manuscript. BL participated in the collection of clinical data and strains from the patient. AG-L has been involved in the antifungal susceptibility testing. IC has made contributions to the analysis of the results. MC-E has been involved in drafting the manuscript and in the final approval of the version to be published following a critical review thereof. MJB was responsible for the original design of the study and participated in its further design and development as well as having been involved in drafting the manuscript. All authors have read and approved the final manuscript.

\section{Acknowledgements}

This work was supported by Research Projects from Spanish Fondo de Investigaciones Sanitarias of the Instituto de Salud Carlos III (PI09/1791 and PI11/00412) and by the Spanish Network for Research on Infectious Diseases (REIPI RD06/0008/10). S. G. is supported by a research fellowship from the "Fondo de Investigaciones Biomedicas" of the Spanish Ministry of Science and Innovation (FI10/00464).

\section{Author details}

'Servicio de Micología, Centro Nacional de Microbiología, Instituto de Salud Carlos III, Ctra Majadahonda-Pozuelo, Km 2, Majadahonda, Madrid 28220, Spain. ${ }^{2}$ Servicio de Microbiología, Hospital Virgen de la Concha, Zamora, España. ${ }^{3}$ Unidad de Bioinformática, Centro Nacional de Microbiología, Instituto de Salud Carlos III, Madrid, Spain.

Received: 23 January 2012 Accepted: 18 January 2013 Published: 23 January 2013

\section{References}

1. Pappas PG: Invasive candidiasis. Infect. Dis Clin North Am 2006, 20:485-506

2. Khatib R, Ayeni O, Riederer KM, Briski LE, Wilson FM: Strain relatedness in persistent and recurrent candiduria. J Urol 1998, 159:2054-2056.

3. Lockhart SR, Fritch JJ, Meier AS, Schroppel K, Srikantha T, Galask R, Soll DR: Colonizing populations of Candida albicans are clonal in origin but undergo microevolution through $\mathrm{C} 1$ fragment reorganization as demonstrated by DNA fingerprinting and $\mathrm{C} 1$ sequencing. J Clin Microbiol 1995, 33:1501-1509.

4. Lockhart SR, Reed BD, Pierson CL, Soll DR: Most frequent scenario for recurrent Candida vaginitis is strain maintenance with "substrain shuffling": demonstration by sequential DNA fingerprinting with probes Ca3, C1, and CARE2. J Clin Microbiol 1996, 34:767-777.

5. Da Matta DA, Melo AS, Guimaraes T, Frade JP, Lott TJ, Colombo AL: Multilocus sequence typing of sequential Candida albicans isolates from patients with persistent or recurrent fungemia. Med Mycol 2010, 48:757-762.

6. Jacobsen MD, Duncan AD, Bain J, Johnson EM, Naglik JR, Shaw DJ, Gow NA, Odds FC: Mixed Candida albicans strain populations in colonized and infected mucosal tissues. FEMS Yeast Res 2008, 8:1334-1338.
7. Odds FC, Davidson AD, Jacobsen MD, Tavanti A, Whyte JA, Kibbler CC, Ellis DH, Maiden MC, Shaw DJ, Gow NA: Candida albicans strain maintenance, replacement, and microvariation demonstrated by multilocus sequence typing. J Clin Microbiol 2006, 44:3647-3658.

8. Sabino R, Sampaio P, Carneiro C, Rosado L, Pais C: Isolates from hospital environments are the most virulent of the Candida parapsilosis complex. BMC Microbiol 2011, 11:180.

9. Sampaio P, Gusmao L, Correia A, Alves C, Rodrigues AG, Pina-Vaz C, Amorim A, Pais C: New microsatellite multiplex PCR for Candida albicans strain typing reveals microevolutionary changes. J Clin Microbiol 2005, 43:3869-3876.

10. Shin JH, Chae MJ, Song JW, Jung SI, Cho D, Kee SJ, Kim SH, Shin MG, Suh SP, Ryang DW: Changes in karyotype and azole susceptibility of sequential bloodstream isolates from patients with Candida glabrata candidemia. J Clin Microbiol 2007, 45:2385-2391.

11. Shin JH, Park MR, Song JW, Shin DH, Jung SI, Cho D, Kee SJ, Shin MG, Suh SP, Ryang DW: Microevolution of Candida albicans strains during catheter-related candidemia. J Clin Microbiol 2004, 42:4025-4031.

12. Sampaio P, Santos M, Correia A, Amaral FE, Chavez-Galarza J, Costa-deOliveira S, Castro AG, Pedrosa J, Pais C: Virulence attenuation of Candida albicans genetic variants isolated from a patient with a recurrent bloodstream infection. PLoS One 2010, 5:e10155.

13. Huang M, McClellan M, Berman J, Kao KC: Evolutionary dynamics of Candida albicans during in vitro evolution. Eukaryot Cell 2011, 10:1413-1421.

14. Botterel F, Desterke C, Costa C, Bretagne S: Analysis of microsatellite markers of Candida albicans used for rapid typing. J Clin Microbiol 2001, 39:4076-4081.

15. Costa JM, Garcia-Hermoso D, Olivi M, Cabaret O, Farrugia C, Lecellier G, Dromer F, Bretagne S: Genotyping of Candida albicans using length fragment and high-resolution melting analyses together with minisequencing of a polymorphic microsatellite locus. J Microbiol Methods 2010, 80:306-309.

16. Costa-de-Oliveira S, Sousa I, Correia A, Sampaio P, Pais C, Rodrigues AG, Pina-Vaz C: Genetic relatedness and antifungal susceptibility profile of Candida albicans isolates from fungaemia patients. Med Mycol 2011, 49:248-252

17. Eloy O, Marque S, Botterel F, Stephan F, Costa JM, Lasserre V, Bretagne S: Uniform distribution of three Candida albicans microsatellite markers in two French ICU populations supports a lack of nosocomial crosscontamination. BMC Infect Dis 2006, 6:162.

18. Garcia-Hermoso D, Cabaret O, Lecellier G, Desnos-Ollivier M, Hoinard D, Raoux D, Costa JM, Dromer F, Bretagne S: Comparison of microsatellite length polymorphism and multilocus sequence typing for DNA-Based typing of Candida albicans. J Clin Microbiol 2007, 45:3958-3963.

19. Garcia-Hermoso D, MacCallum DM, Lott TJ, Sampaio P, Serna MJ, Grenouillet F, Klaassen CH, Bretagne S: Multicenter collaborative study for standardization of Candida albicans genotyping using a polymorphic microsatellite marker. J Clin Microbiol 2010, 48:2578-2581.

20. Erali M, Voelkerding KV, Wittwer CT: High resolution melting applications for clinical laboratory medicine. Exp Mol Pathol 2008, 85:50-58.

21. Erali $M$, Wittwer $C T$ : High resolution melting analysis for gene scanning. Methods 2010, 50:250-261.

22. Arancia S, Sandini S, De Bernardis F, Fortini D: Rapid, simple, and low-cost identification of Candida species using high-resolution melting analysis. Diagn Microbiol Infect Dis 2011, 69:283-285.

23. Rodriguez-Tudela JL Arendrup MC, Barchiesi F, Bille J, Chryssanthou E, Cuenca-Estrella M, Dannaoui E, Denning DW, Donnelly JP, Dromer F, Fegeler W, Lass-Flörl C, Moore C, Richardson M, Sandven P, Velegraki A, Verweij P: EUCAST definitive document EDef 7.1: method for the determination of broth dilution MICs of antifungal agents for fermentative yeasts. Clin Microbiol Infect 2008, 14:398-405.

24. Rodriguez-Tudela JL, Arendrup MC, Cuenca-Estrella M, Donnelly JP, LassFlörl C: EUCAST Breakpoints for Antifungals. Drugs News and Perspectives 2010, 23:93-97.

25. Schoofs A, Odds FC, Colebunders R, leven M, Wouters L, Goossens H: Isolation of Candida species on media with and without added fluconazole reveals high variability in relative growth susceptibility phenotypes. Antimicrob Agents Chemother 1997, 41:1625-1635.

26. Cendejas-Bueno E, Gomez-Lopez A, Mellado E, Rodriguez-Tudela JL, Cuenca-Estrella M: Identification of pathogenic rare yeast species in 
clinical samples: comparison between phenotypical and molecular methods. J Clin Microbiol 2010, 48:1895-1899.

27. Tang CM, Cohen J, Holden DW: An Aspergillus fumigatus alkaline protease mutant constructed by gene disruption is deficient in extracellular elastase activity. Mol Microbiol 1992, 6:1663-1671.

28. DiDomenico BJ, Brown NH, Lupisella J, Greene JR, Yanko M, Koltin Y Homologs of the yeast neck filament associated genes: isolation and sequence analysis of Candida albicans CDC3 and CDC10. Mol Gen Genet 1994, 242:689-698.

29. Bretagne S, Costa JM, Besmond C, Carsique R, Calderone R: Microsatellite polymorphism in the promoter sequence of the elongation factor 3 gene of Candida albicans as the basis for a typing system. J Clin Microbiol 1997, 35:1777-1780

30. Magee BB, Koltin Y, Gorman JA, Magee PT: Assignment of cloned genes to the seven electrophoretically separated Candida albicans chromosomes. Mol Cell Biol 1988, 8:4721-4726.

31. Hunter PR, Gaston MA: Numerical index of the discriminatory ability of typing systems: an application of Simpson's index of diversity. J Clin Microbiol 1988, 26:2465-2466.

32. Myoung Y, Shin JH, Lee JS, Kim SH, Shin MG, Suh SP, Ryang DW: Multilocus sequence typing for Candida albicans isolates from candidemic patients: comparison with Southern blot hybridization and pulsed-field gel electrophoresis analysis. Korean J Lab Med 2011, 31:107-114.

33. Cartledge JD, Midgley J, Gazzard BG: Clinically significant azole crossresistance in Candida isolates from HIV-positive patients with oral candidosis. AIDS 1997, 11:1839-1844.

34. Johnson EM, Warnock DW, Luker J, Porter SR, Scully C: Emergence of azole drug resistance in Candida species from HIV-infected patients receiving prolonged fluconazole therapy for oral candidosis. $J$ Antimicrob Chemother 1995, 35:103-114.

35. Mader E, Lukas B, Novak J: A strategy to setup codominant microsatellite analysis for high-resolution-melting-curve-analysis (HRM). BMC Genet 2008, 9:69.

36. Wittwer CT, Reed GH, Gundry CN, Vandersteen JG, Pryor RJ: High-resolution genotyping by amplicon melting analysis using LCGreen. Clin Chem 2003, 49:853-860.

doi:10.1186/1471-2180-13-13

Cite this article as: Gago et al:: Analysis of strain relatedness using High Resolution Melting in a case of recurrent candiduria. BMC Microbiology 2013 13:13

\section{Submit your next manuscript to BioMed Central and take full advantage of:}

- Convenient online submission

- Thorough peer review

- No space constraints or color figure charges

- Immediate publication on acceptance

- Inclusion in PubMed, CAS, Scopus and Google Scholar

- Research which is freely available for redistribution 